\title{
SPubínSaúde
}

\section{Abordagem inicial em neonatologia na presença de erro inato do metabolismo}

Initial approach in neonatology in the presence of an inborn metabolism error

\author{
Bruno César Fernandes ${ }^{*} \bullet$, Alan Márcio de Brito Araújo ${ }^{1} \bullet$, Nédson Lechner da Silva ${ }^{\oplus}$, Mariella \\ Rodrigues da Silva ${ }^{1}{ }^{\circ}$, Raquel Borges de Barros Primo ${ }^{2} \bullet$
}

${ }^{1}$ Hospital Universitário da Universidade Federal Grande Dourados, Ebserh, Dourados, Mato Grosso do Sul, Brasil. ${ }^{2}$ Programa de PósGraduação em Ciências da Saúde. Faculdade de Ciências da Saúde, Universidade Federal da Grande Dourados, Dourados, Mato Grosso do Sul, Brasil.*Autor para correspondência. E-mail: brunoanaisafernandes@gmail.com.

\begin{abstract}
Resumo: Introdução: Os erros inatos do metabolismo (EIM) são doenças hereditárias de um único gene que resultam em defeitos nas vias bioquímicas do corpo. Embora esses distúrbios sejam individualmente raros, coletivamente são responsáveis por uma parcela significativa das incapacidades e mortes na infância. Revisão: Profissionais da área de saúde ainda encontram certa dificuldade na identificação precoce dos casos dessas doenças e no estabelecimento de uma terapêutica apropriada. Assim, o objetivo do estudo é compreender como deve ser a abordagem inicial em neonatologia quando se identifica um EIM em um paciente. A metodologia utilizada no desenvolvimento desse estudo foi a revisão da literatura, de caráter qualitativo. Discussão: Foram selecionadas 13 referências, sendo 10 artigos científicos, um Manual Técnico do Ministério da Saúde e duas reportagens sobre Teste do Pezinho expandido. No total, oito são no idioma português e cinco no idioma inglês. Considerações finais: Os resultados da pesquisa permitiram constatar que diante da suspeita de um EIM a equipe de saúde neonatal deve seguir o seguinte protocolo: executar o teste de diagnóstico; parar o catabolismo fornecendo glicose; administrar cuidados de suporte; remover metabólitos tóxicos; considerar sepse (pois essa pode imitar a doença metabólica). Abordagens de tratamento como dietas especiais, terapia de reposição enzimática, inibição de substrato e transplante de órgãos têm sido amplamente utilizadas. É fundamental, portanto, que a equipe de saúde neonatal esteja capacitada e atualizada sobre as inovações nessa área, para poder fornecer ao paciente a melhor opção de cuidado em saúde.
\end{abstract}

Palavras-chave: erro inato do metabolismo, neonato, teste de triagem neonatal.

\begin{abstract}
Introduction: Inborn errors of metabolismo (EIM) are inherited diseases of a single gene that result in defects in the body's biochemical pathways. Although these disorders are individually rare, collectively they are responsible for a significant portion of childhood disabilities and deaths. Review: Health care professionals still find it difficult to identify cases of these diseases early and to establish appropriate therapy. Thus, the aim of the study is to understand how the initial approach in neonatology should be when identifying an IMT in a patient. The methodology used in the development of this study was a qualitative review of the literature. Discussion: 13 references were selected, 10 of which were scientific articles, a Technical Manual from the Ministry of Health and two reports on the expanded Pezinho test. In total, eight are in Portuguese and five in English. Final considerations: The results of the research showed that, in the event of a suspicion of an EIM, the neonatal health team must follow the following protocol: perform the diagnostic test; stop catabolism by providing glucose; administer supportive care; remove toxic metabolites; consider sepsis (as this can mimic metabolic disease). Treatment approaches such as special diets, enzyme replacement therapy, substrate inhibition and organ transplantation have been widely used. It is essential, therefore, that the neonatal health team is trained and updated on innovations in this area, in order to provide the patient with the best option of health care.
\end{abstract}

Keywords: inborn error of metabolism, neonate, neonatal screening test.

\section{Introdução}

Os erros inatos do metabolismo (EIM) são desordens de gene único que resultam em defeitos nas vias bioquímicas do corpo. Embora esses distúrbios sejam individualmente raros, eles, coletivamente, são responsáveis por uma parcela significativa das incapacidades e mortes na infância. A maioria dos distúrbios é herdada como doença autossômica recessiva, mas distúrbios autossômicos dominantes e ligados ao cromossomo X também estão presentes. Os sinais e sintomas clínicos surgem do acúmulo do substrato 
tóxico, deficiência do produto, ou ambos. Dependendo da atividade residual da enzima deficiente, o início do quadro clínico pode variar desde o período neonatal até a idade adulta (Ezgu, 2016).

Os EIM são patologias graves e de difícil reconhecimento. Em geral, seus sintomas iniciais se manifestam em neonatos e em crianças, sendo o diagnóstico precoce muito importante para um manejo adequado do quadro clínico desses pacientes (Azevedo et al., 2017). Centenas de desordens já foram descritas e houve uma considerável sobreposição clínica entre certos erros inatos nos últimos anos. Resultante deste fato, o diagnóstico definitivo de EIM depende de ensaios enzimáticos ou testes genéticos, mas, especialmente durante os últimos anos, realizações significativas foram obtidas para o diagnóstico bioquímico e genético de erros inatos (Ezgu, 2016).

A melhor forma de um diagnóstico precoce é através da triagem neonatal, conhecida popularmente como “teste do pezinho", que é capaz de detectar algumas dessas doenças. A média de idade do diagnóstico de um EIM costuma ocorrer em crianças em torno de 4,3 anos de idade, quando, infelizmente, para algumas crianças, já se encontram instaladas algumas sequelas irreversíveis, mesmo com elas apresentando sintomas antes de um ano de vida (Camelo Junior, 2017).

As doenças causadas por defeitos no metabolismo intermediário de pequenas moléculas em geral possuem apresentação mais precoce, com quadro sintomatológico mais exuberante, e isso costuma permitir um diagnóstico precoce. Também ajuda a conduzir a um tratamento mais imediato possível para ser eficaz na prevenção de danos e para permitir uma melhor qualidade de vida ao paciente, o que pode ser conquistado através de tratamentos dietéticos, reposição de cofatores, transplantes hepáticos, entre outros (Camelo Junior, 2017).

A justificativa pela escolha do tema se fundamenta no fato de que, apesar do conhecimento sobre EIM, nos últimos anos, estar aumentando, os profissionais da área de saúde ainda encontram certa dificuldade na identificação precoce dos casos dessas doenças e no estabelecimento de uma terapêutica apropriada. Portanto, a realização de um estudo que reúna as principais informações recentes sobre o diagnóstico e a abordagem inicial dessas condições é relevante para a comunidade científica e educacional.

Diante do exposto, o objetivo desta pesquisa foi de compreender como deve ser a abordagem inicial em neonatologia quando se identifica um erro inato do metabolismo em um paciente.

\section{Revisão}

A metodologia utilizada no desenvolvimento desse estudo foi a revisão da literatura, optando-se por utilizar apenas os artigos científicos publicados em revistas/periódicos, por trazerem as informações mais atuais, bem como por alguns trazerem dados de estudos clínicos. Para focar apenas em informações mais recentes, o estudo utilizou como critério de escolha para os artigos que estes fossem publicados entre $2015 \mathrm{e}$ 2020. Também foi feita a opção por artigos publicados apenas em português ou inglês.

As bases de dados utilizadas para a pesquisa dos artigos foram: PubMed, Biblioteca Virtual em Saúde (BVS) e Google Acadêmico. Para encontrar os artigos foram utilizadas as seguintes palavras-chave: erro inato do metabolismo; neonato; pediatria; teste de triagem neonatal; teste do pezinho. Na base de dados PubMed foram utilizadas as seguintes palavras-chave: inborn error of metabolism; neonate; pediatrics; neonatal screening test.

Foram selecionadas 13 referências, sendo 10 artigos científicos, um Manual Técnico do Ministério da Saúde e duas reportagens sobre Teste do Pezinho expandido. No total, oito são no idioma português e cinco no idioma inglês.

\section{Discussão}

A morbimortalidade infantil relaciona com EIM geralmente ocorre devido a agravos relacionados à hiperglicemia, hipoglicemia, hipercalcemia, hipocalcemia, hipermagnesemia, hipomagnesemia e hiperbilirrubinemia (Lise, Santos, \& Schwartz, 2017). Essas condições podem ser rastreadas através da triagem neonatal, o que possibilita uma intervenção precoce, visto que a maioria dos neonatos pode não apresentar sintomas ao nascer, podendo se manifestar nas primeiras horas ou mesmo após dias após o parto. Entretanto, quando estão sintomáticos e não são tratados adequadamente, podem apresentar uma evolução negativa, originando déficit neurológico, disfunção hepática, transtornos digestivos, respiratórios, cardiopatia, entre outros problemas (Lise et al., 2019).

O bloqueio da via bioquímica, resultante do defeito genético, provoca acúmulo do substrato que pode diretamente mostrar efeitos tóxicos ou indiretamente pelo desvio do fluxo metabólico para outras vias, bem 
como a deficiência do produto (Ezgu, 2016). Embora individualmente essas doenças sejam relativamente raras, cumulativamente são bastante comuns (Macneill\& Walker, 2018).

Paralelamente ao desenvolvimento de novos métodos diagnósticos, um progresso significativo também foi obtido para o tratamento de várias dessas condições. Abordagens de tratamento como dietas especiais, terapia de reposição enzimática, inibição de substrato e transplante de órgãos têm sido amplamente utilizadas. É óbvio que, com a ajuda da pesquisa pré-clínica e clínica realizada para erros inatos, melhores métodos de diagnóstico e melhores abordagens de tratamento ainda estarão disponíveis (Ezgu, 2016).

A triagem neonatal, é uma importante iniciativa de saúde pública que foi estabelecida como um meio de deteç̧ão precoce e identificação de condições graves em recém-nascidos para os quais existe uma terapia eficaz (Bodamer, Scott, \& Giugliani, 2017). No Brasil, a triagem neonatal consiste em quatro testes: teste do pezinho; teste do olhinho; teste da orelhinha; teste do coraçãozinho (Brasil, 2016). Para o presente estudo, interessa o teste do pezinho, pois é o que detecta os EIM.

A triagem neonatal via teste do pezinho é realizada através de uma punção no calcanhar do recémnascido (RN), por ser esta área bastante vascularizada, sendo o sangue colhido em um papel-filtro, devendo ser realizada entre o terceiro e o sétimo dia de vida. Esse teste permite a identificação de até 30 doenças, sendo que, no Brasil, por meio do Programa Nacional de Triagem Neonatal do Sistema Único de Saúde (SUS), apenas são identificadas o hipotireoidismo congênito, a anemia falciforme, a hiperfenilalaninemia (fenilcetonúria), a hiperplasia adrenal congênita, a fibrose cística e a deficiência da biotinidase (Lise et al., 2019; Brasil, 2016; Oliveira \& Souza, 2017).

Há dois testes de triagem neonatal expandida no país, porém apenas por via particular. Enquanto a básica detecta apenas 6 doenças, as duas versões ampliadas podem detectar de 10 a 48 doenças (Simon, 2018). Entretanto, em consulta ao DLE - Genética Humana e Doenças Raras (DLE, 2020), verifica-se que há disponível no Brasil, na rede particular, um teste expandido que detecta 60.

Os distúrbios metabólicos neonatais causados por EIM podem se manifestar de forma grave, sendo função dos profissionais da saúde que atendem essa população estarem atentos ao surgimento de qualquer agravo sintomatológico, que pode se manifestar precocemente. Nesse cenário, destaca-se a necessidade em identificar os principais distúrbios metabólicos causados pelos EIM que acometem o recém-nascido (RN). Portanto, em relação ao profissional de saúde que atua em neonatologia, cabe a obrigação de manter-se atualizado, mesmo porque é a equipe de saúde neonatal a responsável pelo atendimento ao RN desde o período imediatamente anterior ao parto até o encaminhamento ao alojamento conjunto com sua mãe ou à unidade neonatal, sendo fundamental que sejam avaliadas as condições de saúde tanto materna quanto do RN, sendo necessário que esses profissionais estejam plenamente capacitados para tanto (Lise et al., 2017).

Os EIM representam um desafio único para esses profissionais, pois correspondem a um grande número de doenças individuais, cada uma com seus riscos e tratamentos específicos, sendo individualmente raras e o domínio da fisiopatologia de cada uma é complexo e está fora da abordagem normal da medicina de emergência. Assim, é importante compreender algumas abordagens básicas e terapias relacionadas com esses distúrbios. Pacientes com EIM podem apresentar-se em estado grave, e a falha em considerar o diagnóstico e iniciar a terapia pode levar à morte ou incapacidade permanente dessa criança. Com os avanços médicos modernos, muitas dessas crianças podem prosperar, por isso, cabe a equipe de saúde neonatal ter uma compreensão básica dessas doenças (Macneill \& Walker, 2018).

Deve-se suspeitar de EIM quando na presença de um RN doente, sem causa clara e crianças com anormalidades laboratoriais inesperadas. Independentemente do tipo de EIM, a maioria dos pacientes apresenta sintomas principalmente neurológicos, como: irritabilidade/letargia, vômitos, hipotonia e, às vezes, convulsões. Esses sintomas podem ser secundários a níveis elevados de amônia em decorrência de distúrbios do ciclo da ureia, acidose metabólica profunda causada por acidemias orgânicas ou hipoglicemia decorrente de distúrbios de oxidação de ácidos graxos ou distúrbios do metabolismo de carboidratos. É incomum que um EIM apresente um único sintoma neurológico, porque todas as funções cerebrais são afetadas por desarranjos metabólicos (por exemplo, a hipotonia de uma aminoacidopatia é acompanhada por irritabilidade e letargia). Exceções a essa regra incluem alguns distúrbios convulsivos, como convulsões dependentes de piridoxina (Macneill \& Walker, 2018).

A hiperamonemia como causa do estado mental alterado merece menção especial. $\mathrm{O}$ acúmulo de amônia afeta os sistemas de neurotransmissores, causando danos neurológicos agudos e crônicos. Agudamente, o cérebro sofre morte celular diretamente desencadeada e indiretamente estimula o edema cerebral, que pode se apresentar clinicamente como vômito, letargia, convulsões ou coma. Tanto a hipotermia quanto a hipertermia podem resultar em um diagnóstico digno de consideração, juntamente com sepse, em um neonato irritável com instabilidade de temperatura. Além da ampla gama de distúrbios metabólicos que 
acumulam amônia e causam descompensação neurológica aguda, existem formas "mais leves" dessas doenças que se manifestam com sintomas psiquiátricos episódicos, dificuldades de aprendizado e episódios estrogênicos (Macneill \& Walker, 2018).

Sintomas respiratórios também são comuns, pois pacientes com EIM em crise metabólica apresentam taquipneia por uma de duas razões: 1) a acidose metabólica causa taquipneia, pois os RN têm um sistema de tamponamento renal imaturo, reduzindo sua capacidade de excretar ácido, o que faz com que a compensação respiratória seja um mecanismo essencial para o gerenciamento da acidose, porém, a falta de reserva fisiológica torna esse mecanismo compensatório menos eficaz, especialmente quando o RN fica mais doente; 2) a hiperamonemia pode causar hiperventilação central e taquipneia, embora os achados laboratoriais resultantes sejam mais consistentes com uma alcalose respiratória primária (Macneill \& Walker, 2018).

O vômito é outro sintoma comum da crise metabólica e pode ser secundário ao estresse neurológico ou à intolerância à nutrição, como pode acontecer nos distúrbios do metabolismo de proteínas e carboidratos. A doença hepática é encontrada em muitos dos EIM especialmente aqueles que envolvem metabolismo de glicogênio e tirosinemia. É preciso que a equipe de saúde neonatal tenha em mente que a doença hepática primária causa desequilíbrios metabólicos que podem mimetizar EIM, incluindo galactose na urina, hiperamonemia e ácidos orgânicos anormais no plasma e na urina. Outro sintoma que deve gerar suspeição de EIM é a disfunção cardíaca primária, que é uma apresentação menos comum para a crise metabólica, mas há alguns fatos a serem lembrados. Defeitos de oxidação de ácidos graxos podem causar uma arritmia primária ou anormalidade de condução e a maioria das disritmias cardíacas foi relatada em algum momento com EIM. É mais provável, no entanto, que esses profissionais vejam uma disfunção cardíaca global secundária à acidose e ao estresse metabólico. Assim, é importante saber que a ressuscitação fluídica pode causar problemas se for feita de forma exagerada (Macneill \& Walker, 2018).

Sendo assim, em uma abordagem voltada para a identificação de EIM, além do teste de triagem, é necessário que o pediatra ou neonatologista esteja atento para sintomas relacionados com distúrbios metabólicos mais comuns nesses casos que são: hiperglicemia, hipoglicemia, hipercalcemia, hipocalcemia, hipermagnesemia, hipomagnesemia e hiperbilirrubinemia (Lise et al., 2017).

De acordo com MacNeill e Walker (2018), diante da suspeita de um EIM, deve-se seguir o seguinte protocolo: executar o teste de diagnóstico; parar o catabolismo; administrar cuidados de suporte; remover metabólitos tóxicos; considerar sepse. Para fazer um diagnóstico preciso de uma EIM, as amostras de sangue devem ser colhidas antes de iniciar a terapia, especialmente glicose, porque a administração rapidamente ofusca os achados laboratoriais. Devem ser solicitados os seguintes exames: gases sanguíneos venosos; glicose sanguínea; painel metabólico abrangente; estudos de coagulação; nível de amônia; urinálise; aminoácidos plasmáticos; ácidos orgânicos da urina, ácidos oróticos e aminoácidos; acilcarnitina; substâncias redutoras na urina.

A acidose metabólica pode ser prontamente demonstrada através de medições de gases sanguíneos e eletrólitos e é caracterizada pela diminuição do pH do sangue, diminuição do bicarbonato (HCO3-) e diminuição da PCO2. Especificamente, a acidose metabólica é definida como pH menor que 7,30, PCO2 menor que 30, e HCO3- sérico menor que 15,2. A PCO2 reduzida reflete a resposta respiratória compensatória, confirmando que a acidose não é secundária a insuficiência respiratória, portanto é uma acidose metabólica (Schillaci, Debrosse, \&Mccandless, 2018).

Para parar o catabolismo, é preciso administrar glicose. As crianças com EIM requerem administração de glicose para duas funções: como fonte de energia para a função celular e para bloquear processos catabólicos. Como tal, um simples bolus de glicose é insuficiente e um nível normal de glicose no sangue não deve tranquilizar o provedor de que a glicose exógena é desnecessária. Na verdade, os RN podem precisar de uma quantidade tão alta de glicose que a insulina pode ser necessária para evitar a hiperglicemia, evitando a inanição celular. Além disso, é imperioso lembrar que as crianças são altamente propensas a hipoglicemia devido ao alto uso de glicose (quase o dobro de um adulto), uma resposta subdesenvolvida a baixos níveis glicêmicos (incluindo glicogenólise e gliconeogênese) e menores reservas de glicogênio (Macneill \& Walker, 2018).

Existem dois subgrupos principais de EIM que requerem atenção emergente para a remoção de metabólitos tóxicos: acidúrias orgânicas de cadeia ramificada e desordens do ciclo da ureia. A hiperamonemia deve ser tratada com uma combinação de benzoato de sódio intravenoso (IV) e fenilacetato de sódio IV e cloridrato de arginina. Essa medicameção forma complexos que evitam o ciclo da ureia e permitem a eliminação da amônia na urina. Se o nível de amônia for maior que $500 \mathrm{mmol} / \mathrm{L}$, a hemodiálise 
deve ser iniciada. Importante ressaltar que é a duração da hiperamonemia, não o nível, que causa danos neurológicos devastadores (Macneill \& Walker, 2018).

Por fim, a sepse deve sempre ser considerada na criança doente. Sepse pode imitar a doença metabólica, pode fornecer o ponto de inflexão que leva a uma apresentação emergente em uma criança com um EIM, e as crianças com EIM são mais suscetíveis à infecção (especialmente aquelas com doença hepática). Lembrando que Escherichia coli é uma preocupação em pacientes com galactosemia e antibióticos devem ser iniciados de forma emergente para cobrir essa infecção potencial6. Importante destacar que quando um RN é identificado como portador de EIM, o diagnóstico deve ser confirmado por testes definitivos, que podem envolver testes bioquímicos, testes enzimáticos ou testes moleculares (Vernon, 2015).

\section{Considerações finais}

O diagnóstico dos EIM através da triagem neonatal possibilita uma intervenção precoce de algumas dessas condições, permitindo que cuidados antecipados através de um tratamento adequado reduza as cronicidades dessas doenças e evite consequências deletérias para o crescimento físico e para o desenvolvimento psicomotor da criança. Porém, como nem todas as EIM são detectadas através desse método, é fundamental que a equipe de saúde neonatal esteja atenta para sintomas relacionados com os distúrbios metabólicos mais comuns que são consequências dessas doenças, como a hiperglicemia, a hipoglicemia, a hipercalcemia, a hipocalcemia, a hipermagnesemia, a hipomagnesemia e a hiperbilirrubinemia, e que a maioria dos pacientes com EIM apresenta sintomas neurológicos associados a essas anormalidades laboratoriais inesperadas.

Diante da suspeita de um EIM, a equipe de saúde neonatal deve seguir o seguinte protocolo: executar o teste de diagnóstico; parar o catabolismo fornecendo glicose; administrar cuidados de suporte; remover metabólitos tóxicos; considerar sepse (pois essa pode imitar a doença metabólica). Importante destacar que quando um RN é identificado como portador de EIM, o diagnóstico deve ser confirmado por testes definitivos. É imperativo que a equipe de saúde neonatal se mantenha sempre atualizada e apta para identificar a presença de um EIM em seus pacientes, permitindo o diagnóstico precoce e a instauração do tratamento adequado, evitando que efeitos deletérios dessas doenças venham a interferir não apenas na saúde da criança, mas em seu pleno desenvolvimento no futuro.

\section{Referências}

Azevedo, A. P. G., Cardoso, A. R. D. S., Rosa, A. T. D., Lobato, C. M. D., D’Andréa, L. D. S., Refosco, L. F., ... \& Koladicz, K. R. J. 2017. Resultados de 15 anos de um serviço gratuito para informações e suporte em erros inatos do metabolismo. Clinical and Biomedical Research, 37, 284..

Brasil. 2016.Triagem neonatal biológica: manual técnico. Brasília, DF: Ministério da Saúde.

Bodamer, O. A., Scott, C. R,\&Giugliani, R. 2017. Pompe Disease Newborn Screening Working Group. Newborn Screening for Pompe Disease. Pediatrics, 140(Suppl 1), 4-13.

Camelo Jr, J. S. 2017. Da triagem neonatal expandida à era pós-genômica. Revista Paulista de Pediatria, 35(3), 240-241.

DLE - Genética Humana e Doenças Raras. 2020.Teste do pezinho expandido. Disponível em: https://bit.ly/2xx8RAw

Ezgu, F. 2016. Inborn errors of metabolism. Advances in Clinical Chemistry, 73, 195-250.

Lise, F., Santos, B. P., \& Schwartz, E. 2017. Distúrbios metabólicos no recém-nascido. Revista Espaço Ciência \& Saúde, 5(1), 104-113.

Lise, F., Machado Feijó, A., Marten Milbrath, V., \& Schwartz, E. 2019. Erros inatos do metabolismo do recém-nascido: atualização de enfermagem. Revista Científica de Enfermagem-RECIEN, 9(25).

MacNeill, E. C., \& Walker, C. P. (2018). Inborn errors of metabolism in the emergency department (undiagnosed and management of the known). Emergency Medicine Clinics, 36(2), 369-385.

Oliveira, E. F., \& Souza, A. P. (2017). A importância da realização precoce do teste do pezinho: o papel do enfermeiro na orientação da triagem neonatal. Id on Line Revista de Psicologia, 11(35), 361-378.

Schillaci, L. A. P., DeBrosse, S. D., \& McCandless, S. E. (2018). Inborn errors of metabolism with acidosis: Organic acidemias and defects of pyruvate and ketone body metabolism. Pediatric Clinics, 65(2), 209230. 
Simon J. 2018. Teste do pezinho básico ou ampliado? Entenda a diferença. Revista Crescer online. Disponível em: https://glo.bo/2Ln3AVu

Vernon, H. J. (2015). Inborn errors of metabolism: advances in diagnosis and therapy. JAMA pediatrics, 169(8), 778-782.

\section{Minicurrículo}

Bruno César Fernandes. Enfermeiro Assistêncial da Empresa Brasileira de Serviços Hospirtalares (EBERH/HU-UFGD), mestrando do Programa de Pós-Graduação Stricto Sensu Mestrado Profissional Ensino em Saúde da Universidade Estadual do Mato Grosso do Sul (PPGES-UEMS) polo Dourados/MS. Pós-graduado em Unidade de Terapia Intensiva, Unidade de Terapia Intensiva Neonatal e Pediátrica (Ingá) e, em Saúde da Família.

Alan Márcio de Brito Araújo. Enfermeiro Assistêncial da Empresa Brasileira de Serviços Hospirtalares (EBERH/HU-UFGD). Especialização em Saúde da Família, Terapia Intensiva Adulto, e, Residência Multiprofissional em Neurologia.

Nédson Lechner da Silva. Possui graduação em Negócios Imobiliários e graduação em Enfermagem. Atualmente é Funcionário Público da EBSERH HU/UFGD, ocupando o cargo de Enfermeiro Assistencial, lotado na UTI Adulto. Especialização em Práticas Avançadas de Enfermagem na Atenção Primária.

Mariella Rodrigues da Silva. Enfermeiro Assistêncial da Empresa Brasileira de Serviços Hospirtalares (EBERH/HU-UFGD).

Raquel Borges de Barros Primo. Doutoranda em Ciências da Saúde pela Universidade Federal da Grande Dourados. Mestre em Ciências da Saúde pela Universidade Federal da Grande Dourados. Graduada em Enfermagem. Atualmente é enfermeira assitencial - EBSERH HUPES-UFBA.

Como citar: Fernandes, B.C., Araújo, A.M.B., Silva, N.L., Silva, M.R., \& Primo, R.B.B. 2021.Abordagem inicial em neonatologia na presença de erro inato do metabolismo. Pubsaúde, 5, 131. DOI: https://dx.doi.org/10.31533/pubsaude5.a131

Recebido: 12 fev. 2021.

Revisado e aceito: 1 mar. 2021.

Conflito de interesse: os autores declaram, em relação aos produtos e companhias descritos nesse artigo, não ter interesses associativos, comerciais, de propriedade ou financeiros que representem conflito de interesse.

Licenciamento: Este artigo é publicado na modalidade Acesso Aberto sob a licença Creative Commons Atribuição 4.0 (CC-BY 4.0). 